\title{
Prognostic value of treatment options for extramammary Paget's disease: a SEER database analysis
}

\author{
Xiaobo $\mathrm{Ma}^{1}$, Yongfeng $\mathrm{Li}^{2}$ \\ ${ }^{1}$ Department of General Surgery, the First Hospital of Shanxi Medical University, Taiyuan, China; ${ }^{2}$ Department of Gastrointestinal Surgery, Union \\ Hospital, Tongji Medical College, Huazhong University of Science and Technology, Wuhan, China \\ Contributions: (I) Conception and design: All authors; (II) Administrative support: All authors; (III) Provision of study materials or patients: All \\ authors; (IV) Collection and assembly of data: All authors; (V) Data analysis and interpretation: All authors; (VI) Manuscript writing: All authors; (VII) \\ Final approval of manuscript: All authors. \\ Correspondence to: Yongfeng Li. Department of Gastrointestinal Surgery, Union Hospital, Tongji Medical College, Huazhong University of Science \\ and Technology, Wuhan 430022, China. Email: liyongfeng@hust.edu.cn.
}

Background: Extramammary Paget's disease (EMPD) is a common subtype of Paget's disease. Still, there are lacking reports concerning its clinical features, treatment options, and prognosis.

Methods: The Surveillance, Epidemiology, and End Results (SEER) database was queried for the patients diagnosed with mammary Paget's disease (MPD) or EMPD from 1975 to 2016. Subsequent analysis was conducted to explore incidence rate, tumor characteristics, clinical features, and survival.

Results: A total of 1,848 patients with EMPD and 7,106 patients with MPD were retrieved from the SEER database and included in this study. The demographics of EMPD and MPD were significantly different. Compared with MPD, EMPD had better cancer-specific survival (CSS) but worse overall survival (OS). For EMPD, age $(\mathrm{P}<0.001)$, male $(\mathrm{P}=0.006)$, chemotherapy $(\mathrm{P}=0.002)$, poorly differentiated and undifferentiated grade (both $\mathrm{P}<0.001$ ) and tumor metastasis (regional: $\mathrm{P}=0.019$; distant: $\mathrm{P}<0.001$ ) were independent negative prognostic indicators. Survival analysis revealed that surgery could improve both CSS and OS for EMPD (both $\mathrm{P}<0.001)$. However, neither radiotherapy $(\mathrm{P}=0.013$ and $\mathrm{P}<0.001)$ nor chemotherapy $(\mathrm{P}=0.007$ and $\mathrm{P}<0.001)$ did not exhibit favorable prognostic benefit.

Conclusions: EMPD had distinct clinical features from MPD. Age, gender, chemotherapy, tumor grade and stage are independent prognostic factors for EMPD. While surgery's protective role was supported, radiotherapy and chemotherapy could be unfavorable treatments concerning EMPD prognosis.

Keywords: Extramammary Paget's disease (EMPD); mammary Paget's disease (MPD); Surveillance, Epidemiology, and End Results (SEER)

Submitted Dec 22, 2020. Accepted for publication Apr 16, 2021.

doi: $10.21037 /$ tcr-20-3492

View this article at: https://dx.doi.org/10.21037/tcr-20-3492

\section{Introduction}

Paget's disease includes mammary Paget's disease (MPD) and extramammary Paget's disease (EMPD). Limited to the epidermis, EMPD is a type of intraepidermal adenocarcinoma. Although EMPD is not life-threatening, it is often associated with underlying in situ or invasive carcinoma $(1,2)$. Surgery is a fundamental way to eradicate both MPD and EMPD $(3,4)$. Meanwhile, alternative treatments for Paget's disease, including photodynamic therapy, laser therapy, radiotherapy, topical treatments, chemotherapy, and cell-specific treatments, have also been raised due to the frequent post-surgery recurrence $(5,6)$.

First described by Sir James Paget's in 1874, MPD was a nipple-areola complex disorder characterized by erythematous and eczematous changes (7-9). In contrast, EMPD, as a rare neoplasm, is considered a type of adenocarcinoma originating from the skin or skin 
appendages in areas with apocrine glands (10-12). The anogenital area in the elderly population could be the typical place affected by EMPD (13). Besides, primary locations of EMPD mainly include the vulvar area, perianal region, scrotum, penis, and axillae $(6,14)$.

While the epidemiological features of MPD have been extensively studied, there is lacking studies to summarize the clinicopathological characteristics, treatment options, and prognosis for EMPD (15-17). In this study, the population-based data were analyzed to depict the profiles of EMPD, including its comparison with MPD. Our findings may contribute to the diagnosis, treatments, and prognosis prediction of EMPD. We present the following article in accordance with the STROBE reporting checklist (available at https://dx.doi.org/10.21037/tcr-20-3492).

\section{Methods}

\section{Data collection}

The Surveillance, Epidemiology, and End Results (SEER) database (SEER 18 Regs Research Data + Hurricane Katrina Impacted Louisiana Cases, Nov 2018 Sub (19752016 varying), was queried for all data in our present study. We had obtained permission to obtain the data in the SEER database. Moreover, downloading data from the SEER database did not require informed patient consent and approval from the Institutional review board (IRB) since the data set, which the National Cancer Institute maintained, excluded sensitive patient information. The study was conducted in accordance with the Declaration of Helsinki (as revised in 2013).

\section{Patient selection}

The data of patients diagnosed with Paget's disease, including MPD and EMPD, were excerpted from the SEER database for inclusion. After excluding the diagnosed patients with missing data, patients diagnosed with EMPD or MPD were included in our study (Figure S1).

The diagnosis of patients was retrieved according to the International Classification of Diseases for Oncology (ICD-O) codes as the following: MPD [8540], Paget's disease with infiltrating ductal carcinoma [8541], EMPD [8542], and Paget's disease with intraductal carcinoma [8543]. The data for primary anatomic sites of EMPD patients were extracted according to ICD-O codes (C519 for vulva; C440-C449 for skin; C600-C602, C608-C609, and C632 for penis or scrotum; C510-C512 and C518 for labia; C529 for vagina; and any other coding for other sites). All cases were reconfirmed to have the information concerning the age at diagnosis, tumor size, sex, race, tumor grade, SEER stage, AJCC stage, treatment, and survival. Specifically, due to the SEER stage and AJCC stage share similar properties and many missing values of the AJCC stage, we deleted AJCC stage information and only adopted the SEER historic stage to represent the disease stage for regression analysis.

\section{Statistical analysis}

Categorical variables were presented as counts and percentages, while continuous variables were presented as mean \pm standard deviations (SD). Categorical variables were compared by Pearson's chi-square test or Fisher's exact test, and continuous variables were compared by independent samples Student's $t$-test.

Survival analysis was executed by the Kaplan-Meier method. Mantel-Cox log-rank test was used to assess the significance of differences between survival curves. The Cox proportional hazards models were used to calculate hazard ratios (HRs) and their 95\% confidence intervals (CIs) to analyze individual variables concerning overall survival (OS) further. All statistical analyses were realized within SPSS statistical software, version 22.0 (IBM Corp, Armonk, NY, USA). $\mathrm{P}<0.05$ were considered statistically significant.

\section{Results}

\section{Clinicopathological characteristics}

The clinicopathological characteristics of MPD and EMPD patients were summarized in Table 1. The clinicopathological characteristics of EMPD and MPD were significantly different. The age at diagnosis of EMPD $(70.85 \pm 11.84)$ was significantly larger than that of MPD $(61.41 \pm 15.21)(\mathrm{P}<0.001)$. EMPD patients $(69.6 \%)$ had a larger proportion of patients diagnosed at the age $\geq 66$, compared with MPD patients $(42 \%)(\mathrm{P}<0.001)$. Compared with MPD $(2.52 \pm 2.42)$, EMPD $(4.43 \pm 5.06 \mathrm{~cm})$ tended to have a larger tumor size $(\mathrm{P}<0.001)$. It was not surprising to observe that almost all MPD patients (98.4\%) were females, while two-thirds of EMPD patients (66\%) were also females $(\mathrm{P}<0.001)$.

In terms of treatments, more MPD patients received surgery $(95.9 \%$ vs. $80.9 \%)$, chemotherapy $(23.4 \%$ vs. 
Table 1 Patient clinical characteristics of mammary and extramammary Paget disease

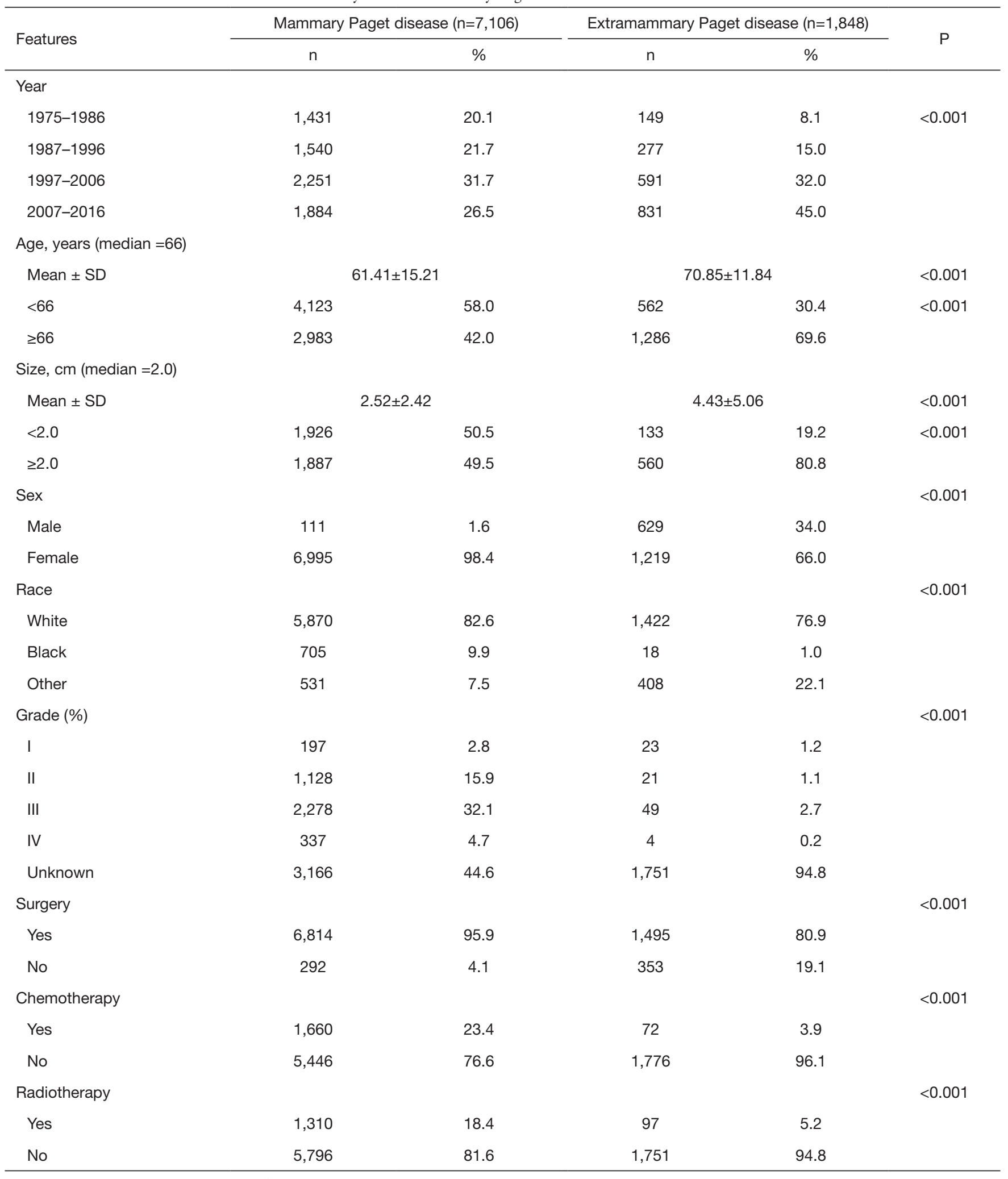

$P$ value $<0.05$ was statistically significant. 

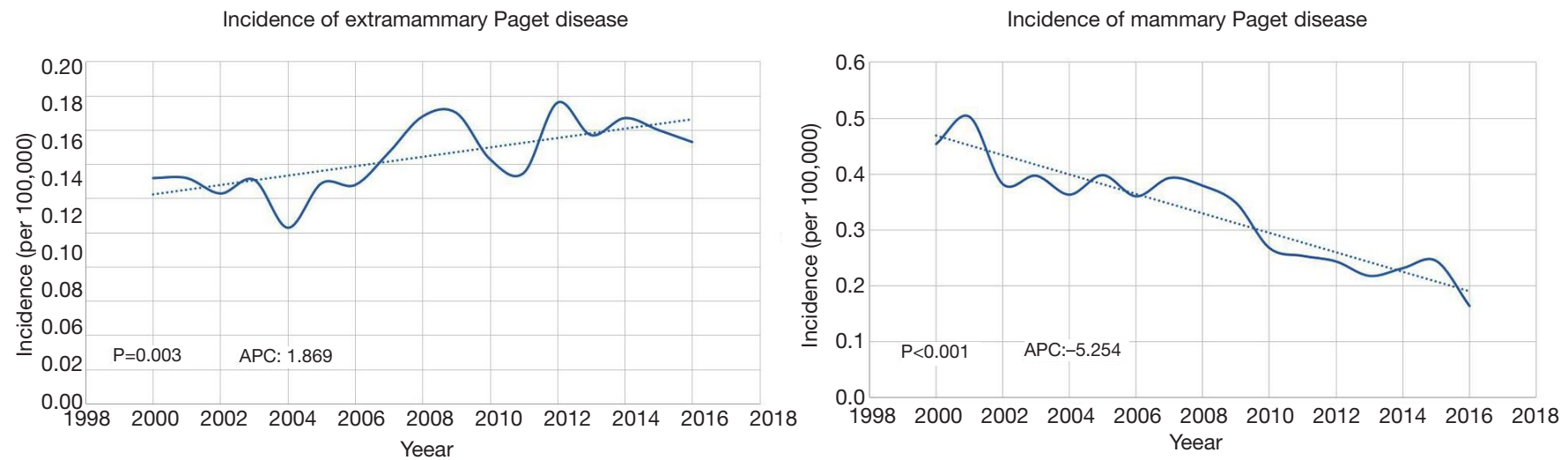

Figure 1 Incidence trend for EMPD (A) and MPD (B) between 2000 and 2016. The solid smooth curve demonstrated the incidence rate and the dotted line could reflect its tendency. EMPD, extramammary Paget's disease; MPD, mammary Paget's disease.

$3.9 \%$ ), and radiotherapy (18.4\% vs. $5.2 \%$ ), compared with EMPD patients $(\mathrm{P}<0.001)$. Furthermore, since most EMPD patients' tumor grade was unknown, the percentage for each grade of MPD patients was significantly higher, especially for III grade $(\mathrm{P}<0.001)$.

Tumor stage information of EMPD patients was also explored, although there were great missing TNM staging system values (Table S1). The result showed that most EMPD patients were diagnosed at an early stage, including T1, N0, M0, stage I, and SEER localized stage. We subsequently sub-grouped EMPD cases based on primary skin locations, including the urogenital system, digestive system, and other systems, and discovered that EMPD in the urogenital system (71.48\%) shared a significant proportion (Figure S2).

\section{Incidence analysis}

Interestingly, the incidence of EMPD increased with an annual percent change (APC), reaching $1.869(\mathrm{P}=0.003)$, while the incidence of MPD had a significant decreasing trend with $\mathrm{APC}=-5.254(\mathrm{P}<0.001)$ (Figure 1). Likewise, the proportion of patients diagnosed with MPD remained stable, while the proportion of patients diagnosed with EMPD escalated during 1975-2016 (Table 1, $\mathrm{P}<0.001$ ).

\section{Survival analysis}

As shown in Figure 2, MPD patients had a better OS rate (Figure $2 A, \mathrm{P}<0.001$ ), while EMPD patients had a better cancer-specific survival (CSS) rate (Figure $2 B, \mathrm{P}<0.001$ ).
However, after matching the diagnosed years and ages of MPD and EMPD patients, the OS rate (Figure S3, $\mathrm{P}=0.014$ ) and CSS rate (Figure S3, $\mathrm{P}<0.001$ ) of EMPD patients were significantly better than those of MPD patients.

Furthermore, the univariate and multivariate analyses were conducted to find the prognostic indicators (Table 2). The univariate analysis results showed that surgical treatment was the significantly protective factor for EMPD $[\mathrm{P}<0.001$, HR $(95 \% \mathrm{CI})=0.57(0.48-0.68)]$. In contrast, the significantly negative prognostic factors for EMPD included age [HR $(95 \% \mathrm{CI})=1.10(1.09-1.11)$, $\mathrm{P}<0.001$ ], male [HR (95\% CI) $=1.33(1.15-1.53), \mathrm{P}<0.001$ ], chemotherapy treatment [HR $(95 \% \mathrm{CI})=1.94(1.43-2.64)$, $\mathrm{P}<0.001)$, radiotherapy treatment $[\mathrm{HR}(95 \% \mathrm{CI})=2.02$ (1.55-2.65), $\mathrm{P}<0.001]$, poorly differentiated grade [HR $(95 \% \mathrm{CI})=3.91(2.82-5.42), \mathrm{P}<0.001]$, undifferentiated grade $[$ HR $(95 \% \mathrm{CI})=6.10(1.96-19.00), \mathrm{P}=0.002]$ and SEER historic stage. The multivariate analysis was then conducted to adjust for confound factors and revealed that the mortality of EMPD patients who are elderly [HR $(95 \%$ $\mathrm{CI})=1.10(1.09-1.11), \mathrm{P}<0.001]$, male $[\mathrm{HR}(95 \% \mathrm{CI})=1.22$ (1.06-1.41), $\mathrm{P}=0.006$ ], with chemotherapy [HR (95\% CI) $=1.67(1.22-2.28), \mathrm{P}=0.002]$, poorly differentiated $[\mathrm{HR}(95 \%$ $\mathrm{CI})=2.36(1.68-3.31), \mathrm{P}<0.001]$ or undifferentiated grade $[$ HR $(95 \% \mathrm{CI})=8.01(2.50-25.69), \mathrm{P}<0.001]$ and SEER distant stage [regional: HR $(95 \% \mathrm{CI})=1.26(1.04-1.52)$, $\mathrm{P}=0.019$; distant: $\mathrm{HR}(95 \% \mathrm{CI})=3.71(2.55-5.39), \mathrm{P}<0.001]$ was significantly higher. Thus, age, gender, chemotherapy, grade and SEER historic stage could serve as independent prognostic factors for EMPD patients.

Lastly, after adjusting the diagnosed years, ages, and 

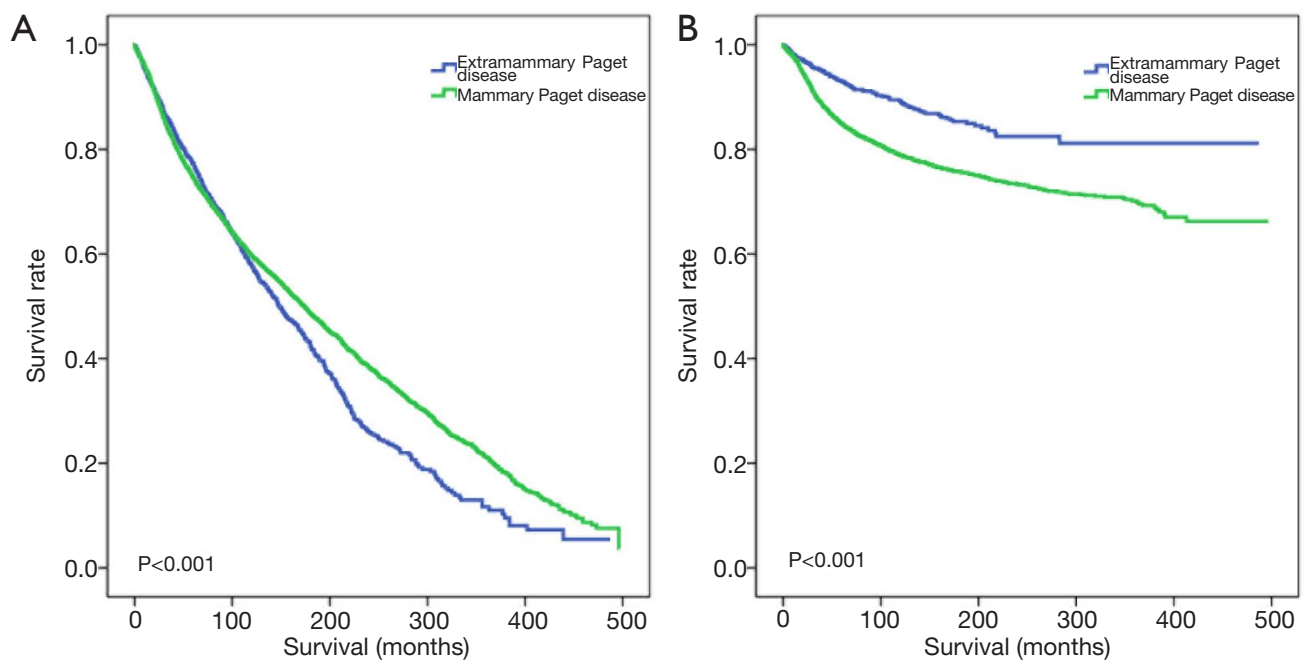

Figure 2 Overall survival (A) and cancer-specific survival (B) for EMPD and MPD. EMPD, extramammary Paget's disease; MPD, mammary Paget's disease.

SEER historic stages of EMPD patients, the survival analyses based on OS and CSS confirmed that EMPD patients could benefit from surgical treatment $(\mathrm{P}<0.001)$ but not chemotherapy $(\mathrm{P}<0.001)$ and radiotherapy $(\mathrm{P}<0.001)$ (Figure 3).

\section{Discussion}

EMPD was a rare disease with morbidity ranging from 0.1 to 2.4 patients per million person-years and commonly affected patients over 65 years old $(18,19)$. While the origin of EMPD was still debatable, EMPD mainly affected those areas with a high apocrine gland concentration, such as genital skin, axillae, and anus (20). Shepherd V had reported that EMPD, which was more common in elderly females, mainly arose in the anogenital area (12). EMPD once seemed to occur more frequently in Asians than in Westerners, with the respective incidence rate of 10 cases per million and 0.9 cases per million (21). However, unlike the previous study, our results discovered that EMPD was more likely to arise in the white population (Table 1). This study revealed that the incidence of EMPD had an increasing trend with an APC $=1.869$ in recent decades (Figure 1). We also confirmed that EMPD was more common in females and the urogenital system (Figure S2). These findings could help profile patient characteristics of EMPD.

Due to its mild progression, EMPD was often found and considered carcinoma in situ with a favorable prognosis (22). Although EMPD is usually confined to the epidermis, it could invade the dermis and metastasize via the lymphatic system as well, during which the prognosis often depends on the depth of invasion (2). Our results showed that the majority of EMPD patients had low tumor stages (Table S1). It would be meaningful to explore whether EMPD patients also had better survival than MPD patients. Although subsequent analysis of CSS supported such hypothesis, survival analyses indicated that the OS of EMPD was significantly worse than that of MPD. Karam et al. once reported that diagnosed age was positively associated with mortality for EMPD patients (23). Therefore, the poor OS for EMPD patients could be attributed to the higher proportion of patients with elder age and lower proportion of patients who underwent surgery (Table 1), which is also supported by the univariate and multivariate analysis study (Table 2).

The best choice for treating EMPD was complete surgical removal in the localized stage $(24,25)$. Specifically, as the gold-standard surgical treatment for certain skin cancers, Mohs micrographic surgery was often used to treat EMPD and had a low local recurrence rate (26). This study reconfirmed the protective role of surgery in treating EMPD that patients who underwent surgical treatment had better OS and CSS. Moreover, surgical treatment was also a favorable prognostic factor (Figure 3).

Furthermore, radiotherapy and chemotherapy were 
Table 2 The univariate and multivariate analysis for extramammary Paget's disease patients based on OS

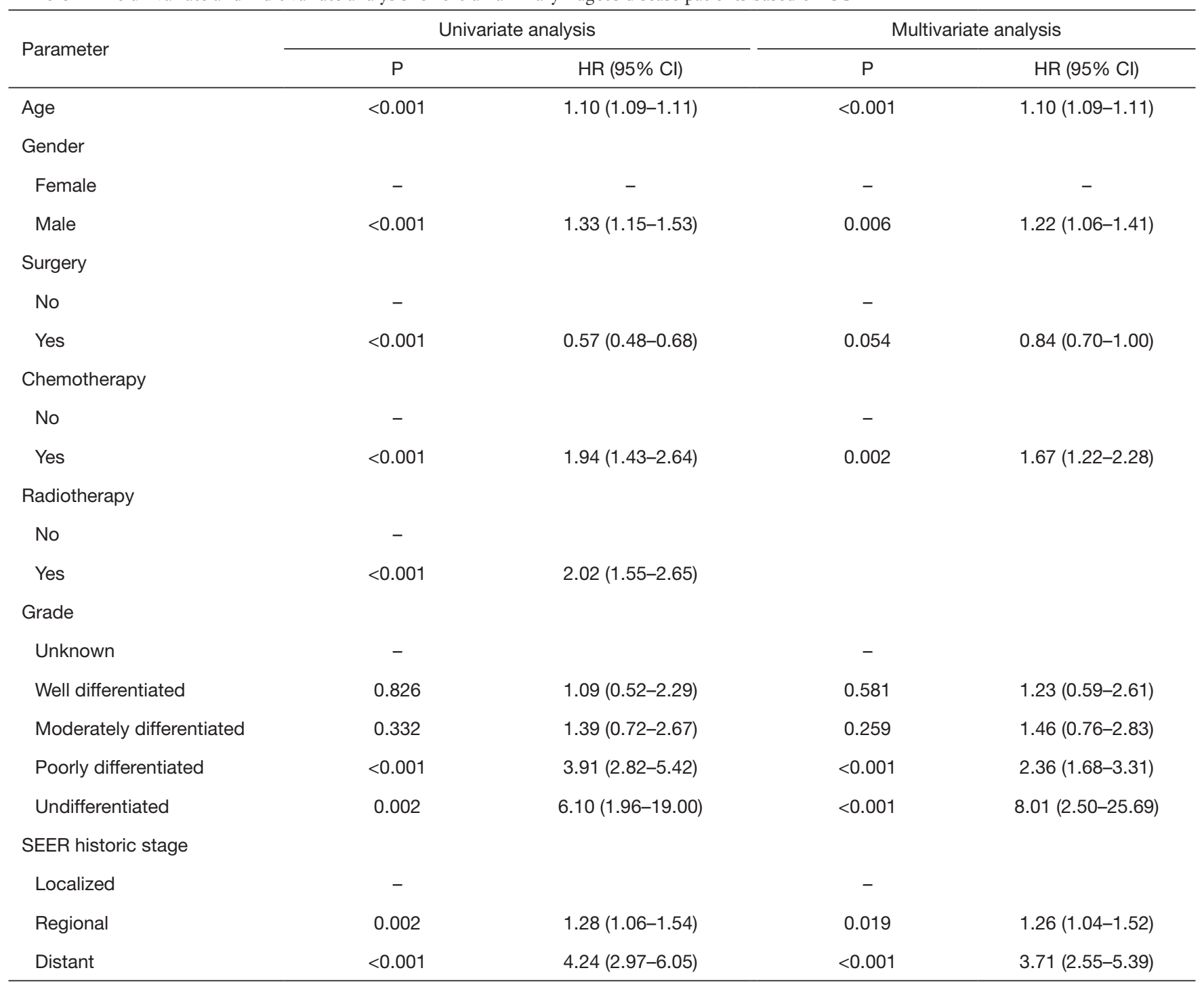

$\mathrm{P}<0.05$ was considered to be significant. OS, overall survival; HR, hazard ratio; Cl, confidence interval; SEER, Surveillance, Epidemiology, and End Results.

once considered to be effective treatments for EMPD due to their successful outcomes (27). However, in this study, radiotherapy and chemotherapy were two negative prognostic factors. Such conflict may attribute to the use of radiotherapy and chemotherapy among the elderly and patients with other severe co-existing diseases, for whom it would be challenging to perform radical surgeries. EMPD patients receiving multiple treatments were not excluded, which would also make the result deviated. Therefore, although our results did not find survival benefits brought by radiotherapy and chemotherapy, they may serve as potential options for EMPD patients, especially when radical resection was not applicable.

Admittedly, there were still limitations to this study. First, only $3.9 \%$ and $2.9 \%$ of patients with EMPD underwent chemotherapy and radiotherapy, which could weaken the final results' statistical power. Second, survival analysis should be interpreted with caution since the EMPD and MPD groups' baseline were significantly different. Patients who underwent surgery may have less advanced tumors, and patients with unresectable advanced tumors are unlikely to receive surgery. Third, this study was retrospective, and prospective exploration is still needed to validate our results. Besides, the SEER historic stage's utilization in this study 

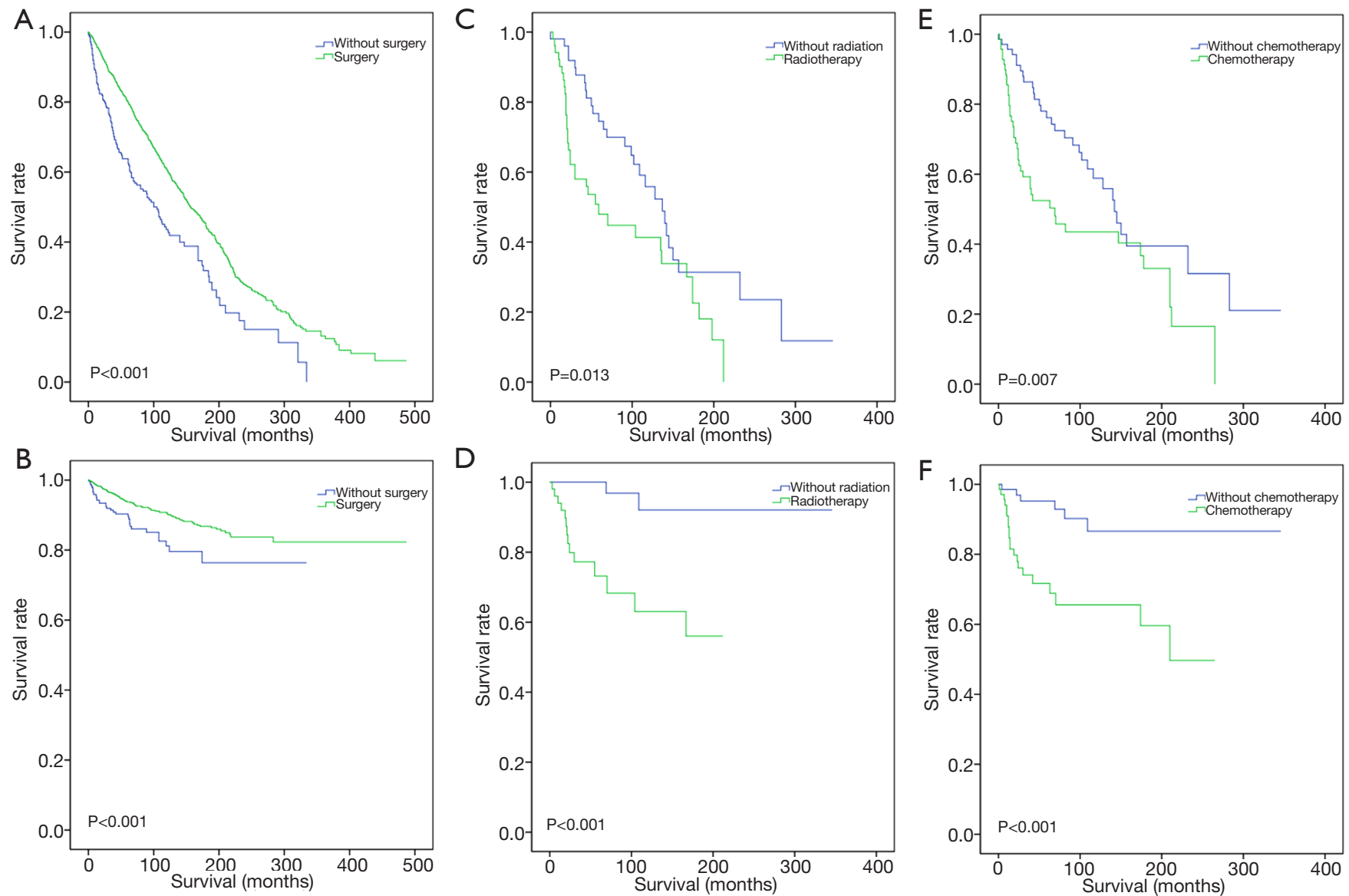

Figure 3 Overall survival (A) and cancer-specific survival (B) with or without surgical treatment for EMPD; overall survival (C) and cancer-specific survival (D) with or without radiotherapy for EMPD; overall survival (E) and cancer-specific survival (F) with or without chemotherapy for EMPD. The diagnosed years, ages, and SEER historic stages of EMPD patients in two compared groups were matched. EMPD, extramammary Paget's disease; SEER, Surveillance, Epidemiology, and End Results.

could be disputable, although there is no EMPD-specific AJCC stage. Notably, an EMPD-specific staging system was once proposed based on primary tumor and metastasis status, but further validation in a larger population is still warranted (28).

Still, this study summarized the clinicopathological characteristics of EMPD, which were significantly different from that of MPD. For EMPD, age, gender, chemotherapy, tumor grade and stage are independent prognostic factors. Our work also supported surgery's protective role, rather than chemotherapy or radiotherapy, in treating EMPD

\section{Acknowledgments}

Funding: None.

\section{Footnote}

Reporting Checklist: Both authors have completed the STROBE reporting checklist. Available at https://dx.doi. org/10.21037/tcr-20-3492

Conflicts of Interest: Both authors have completed the ICMJE uniform disclosure form (available at https://dx.doi. org/10.21037/tcr-20-3492). The authors have no conflicts of interest to declare.

Ethical Statement: The authors are accountable for all aspects of the work in ensuring that questions related to the accuracy or integrity of any part of the work are appropriately investigated and resolved. The study was conducted in accordance with the Declaration of Helsinki (as 
revised in 2013).

Open Access Statement: This is an Open Access article distributed in accordance with the Creative Commons Attribution-NonCommercial-NoDerivs 4.0 International License (CC BY-NC-ND 4.0), which permits the noncommercial replication and distribution of the article with the strict proviso that no changes or edits are made and the original work is properly cited (including links to both the formal publication through the relevant DOI and the license). See: https://creativecommons.org/licenses/by-nc-nd/4.0/.

\section{References}

1. Merritt BG, Degesys CA, Brodland DG. Extramammary Paget Disease. Dermatol Clin 2019;37:261-7.

2. Hartman R, Chu J, Patel R, et al. Extramammary Paget disease. Dermatol Online J 2011;17:4.

3. Wollina U, Goldman A, Bieneck A, et al. Surgical Treatment for Extramammary Paget's Disease. Curr Treat Options Oncol 2018;19:27.

4. Li YJ, Huang XE, Zhou XD. Local Breast Cancer Recurrence after Mastectomy and Breast-Conserving Surgery for Paget's Disease: A Meta-Analysis. Breast Care (Basel) 2014;9:431-4.

5. Nardelli AA, Stafinski T, Menon D. Effectiveness of photodynamic therapy for mammary and extra-mammary Paget's disease: a state of the science review. BMC Dermatol 2011;11:13.

6. Edey KA, Allan E, Murdoch JB, et al. Interventions for the treatment of Paget's disease of the vulva. Cochrane Database Syst Rev 2013;(10):CD009245.

7. Lopes Filho LL, Lopes IM, Lopes LR, et al. Mammary and extramammary Paget's disease. An Bras Dermatol 2015;90:225-31.

8. Karakas C. Paget's disease of the breast. J Carcinog 2011;10:31.

9. Berg JW, Hutter RV. Breast cancer. Cancer 1995;75:257-69.

10. Simonds RM, Segal RJ, Sharma A. Extramammary Paget's disease: a review of the literature. Int J Dermatol 2019;58:871-9.

11. Hatta N. Extramammary Paget's disease. Gan To Kagaku Ryoho 2006;33:1404-7.

12. Shepherd V, Davidson EJ, Davies-Humphreys J. Extramammary Paget's disease. BJOG 2005;112:273-9.

13. Ito T, Kaku Y, Nagae K, et al. Tumor thickness as a prognostic factor in extramammary Paget's disease. J Dermatol 2015;42:269-75.

14. McDaniel B, Brown F, Crane JS. (2020). Extramammary Paget Disease. StatPearls. (Treasure Island (FL).

15. Yao H, Xie M, Fu S, et al. Survival analysis of patients with invasive extramammary Paget disease: implications of anatomic sites. BMC Cancer 2018;18:403.

16. Zhao Y, Sun HF, Chen MT, et al. Clinicopathological characteristics and survival outcomes in Paget disease: a SEER population-based study. Cancer Med 2018;7:2307-18.

17. Sisti A, Huayllani MT, Restrepo DJ, et al. Paget disease of the breast: A national retrospective analysis of the US population. Breast Dis 2020;39:119-26.

18. Herrel LA, Weiss AD, Goodman M, et al. Extramammary Paget's disease in males: survival outcomes in 495 patients. Ann Surg Oncol 2015;22:1625-30.

19. Sopracordevole F, Di Giuseppe J, De Piero G, et al. Surgical Treatment of Paget Disease of the Vulva: Prognostic Significance of Stromal Invasion and Surgical Margin Status. J Low Genit Tract Dis 2016;20:184-8.

20. Lam $\mathrm{C}$ and Funaro D. Extramammary Paget's disease: Summary of current knowledge. Dermatol Clin 2010;28:807-26.

21. Ito T, Kaku-Ito Y, Furue M. The diagnosis and management of extramammary Paget's disease. Expert Rev Anticancer Ther 2018;18:543-53.

22. Kanitakis J. Mammary and extramammary Paget's disease. J Eur Acad Dermatol Venereol 2007;21:581-90.

23. Karam A, Dorigo O. Treatment outcomes in a large cohort of patients with invasive Extramammary Paget's disease. Gynecol Oncol 2012;125:346-51.

24. Murata Y, Kumano K. Extramammary Paget's disease of the genitalia with clinically clear margins can be adequately resected with $1 \mathrm{~cm}$ margin. Eur J Dermatol 2005;15:168-70.

25. Sisti A, Tassinari J, Cuomo R, et al. A case of extramammary inguinal Paget disease in a male patient: surgical treatment with an abdominal advancement cutaneous flap. Acta Biomed 2017;88:79-81.

26. Bae JM, Choi YY, Kim H, et al. Mohs micrographic surgery for extramammary Paget disease: a pooled analysis of individual patient data. J Am Acad Dermatol 2013;68:632-7.

27. Tolia M, Tsoukalas N, Sofoudis C, et al. Primary extramammary invasive Paget's vulvar disease: what is the 
standard, what are the challenges and what is the future for radiotherapy? BMC Cancer 2016;16:563.

28. Ohara K, Fujisawa Y, Yoshino K, et al. A proposal for a

Cite this article as: Ma X, Li Y. Prognostic value of treatment options for extramammary Paget's disease: a SEER database analysis. Transl Cancer Res 2021;10(6):2873-2881. doi: $10.21037 /$ tcr-20-3492
TNM staging system for extramammary Paget disease: Retrospective analysis of 301 patients with invasive primary tumors. J Dermatol Sci 2016;83:234-9. 


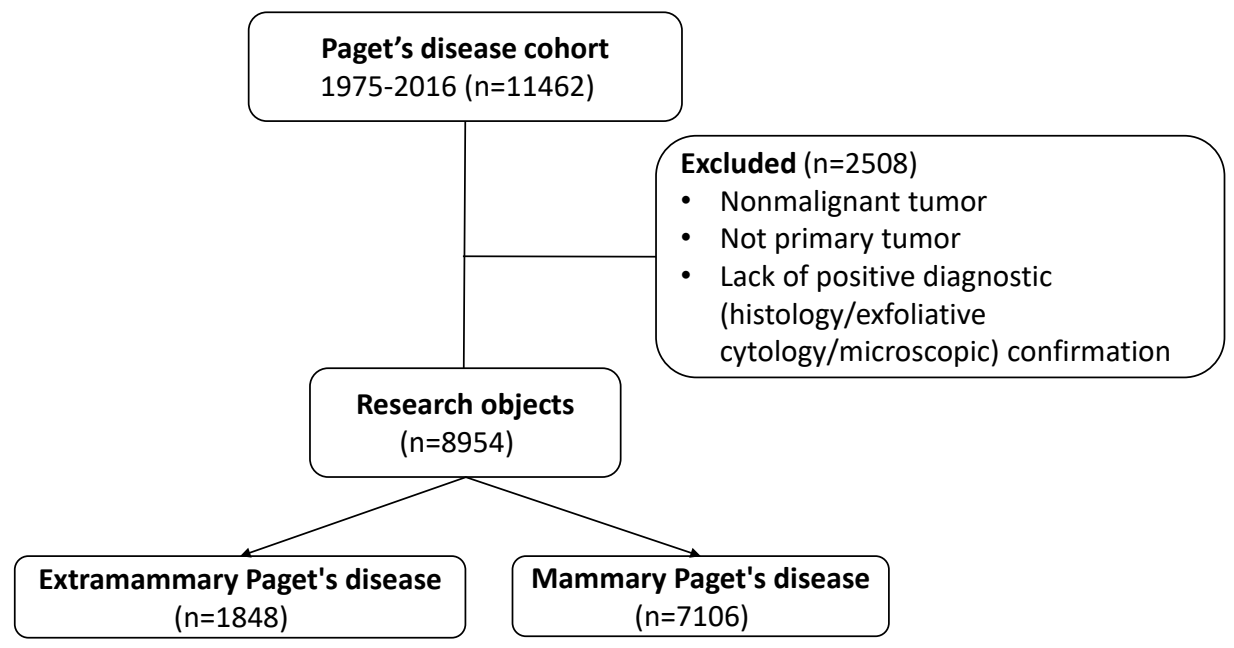

Figure S1 Flow diagram of patient selection.

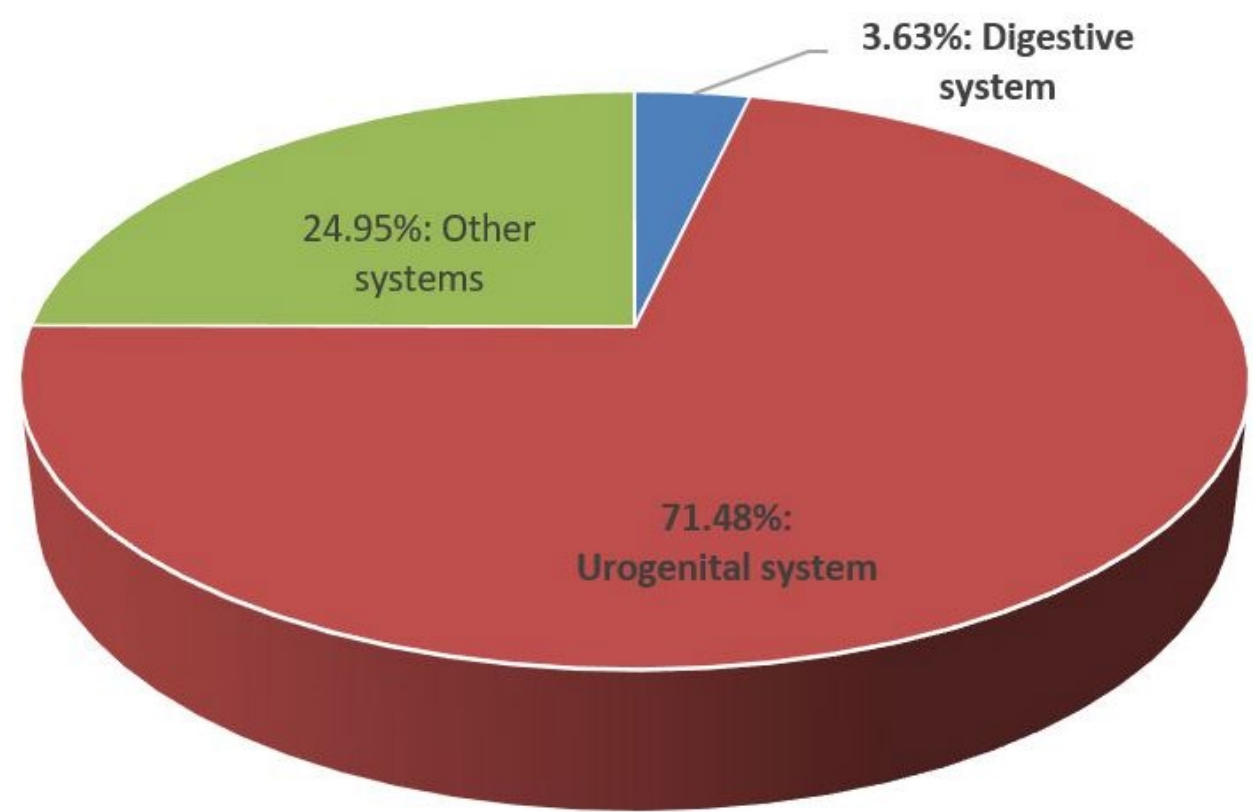

Figure S2 Pie chart to show the proportion of different tumor sites for EMPD. EMPD, extramammary Paget's disease. 
Table S1 TNM, AJCC, and SEER staging for extramammary Paget disease

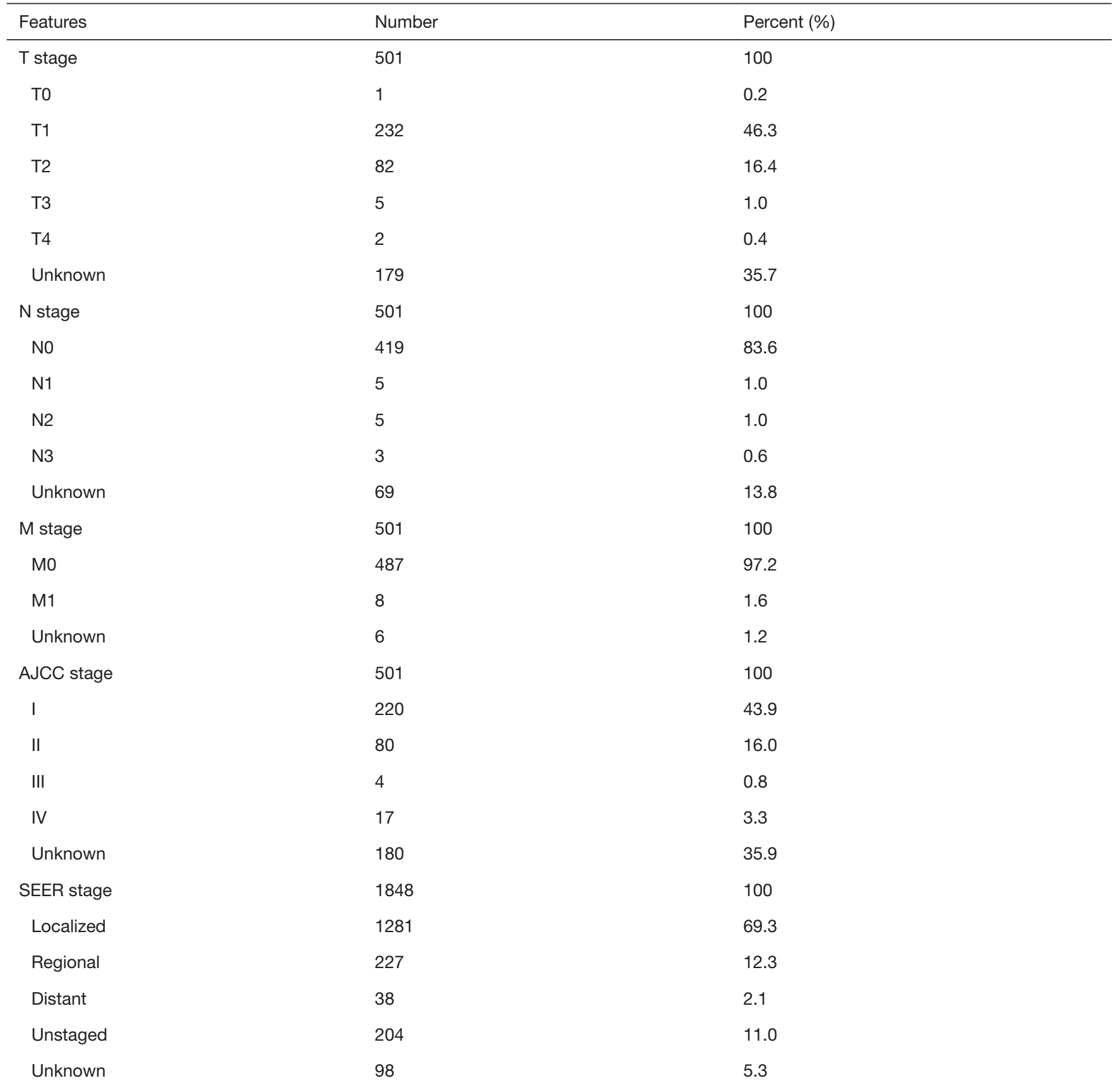

TNM, Tumor, Node, Metastasis; AJCC, American Joint Committee on Cancer; SEER, The Surveillance, Epidemiology, and End Results database. 

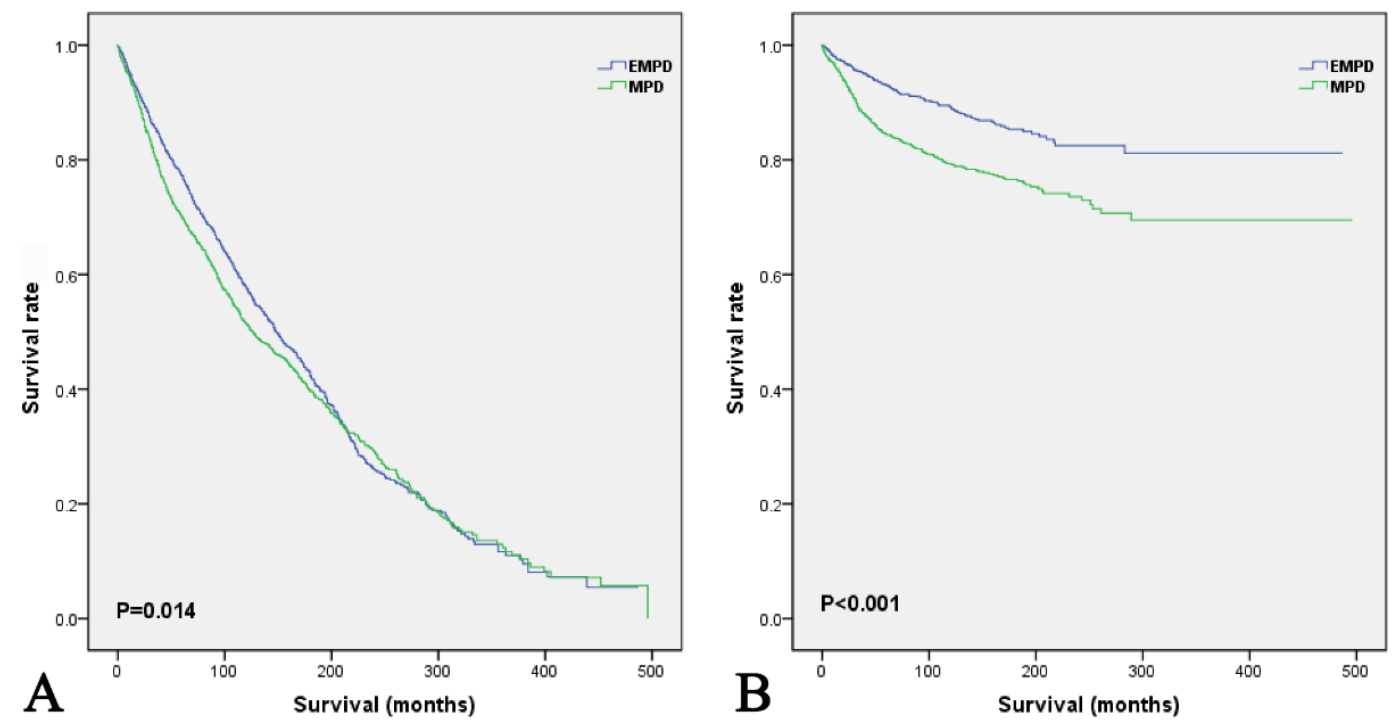

Figure S3 Overall survival (A) and cancer-specific survival (B) for EMPD and MPD. The diagnosed years and ages for MPD and EMPD patients was matched in two compared groups. EMPD, extramammary Paget's disease; MPD, mammary Paget's disease. 